\title{
Decoherent Histories and Hydrodynamic Equations
}

\author{
J.J.HALLIWELL \\ Theory Group \\ Blackett Laboratory \\ Imperial College \\ London \\ SW7 2BZ \\ $U K$ \\ Preprint IC/TP/97-98/50. May, 1998 \\ Submitted to Physical Review D
}

\begin{abstract}
For a system consisting of a large collection of particles, a set of variables that will generally become effectively classical are the local densities (number, momentum, energy). That is, in the context of the decoherent histories approach to quantum theory, it is expected that histories of these variables will be approximately decoherent, and that their probabilites will be strongly peaked about hydrodynamic equations. This possibility is explored for the case of the diffusion of the number density of a dilute concentration of foreign particles in a fluid. This system has the appealing feature that the microscopic dynamics of each individual foriegn particle is readily obtained and the approach to local equilibrium may be seen explicitly. It is shown that, for certain physically reasonable initial states, the probabilities for histories of number density are strongly peaked about evolution according to the diffusion equation. Decoherence of these histories is also shown for a class of initial states which includes non-trivial superpositions of number density. Histories of phase space densities are also discussed. The case of histories of number, momentum and energy density for more general systems, such as a dilute gas, is also discussed in outline. When the initial state is a local equilibrium state, it is shown that the histories are trivally decoherent, and that the probabilities for histories are peaked about hydrodynamic equations. An argument for decoherence of more general initial states is given.
\end{abstract}




\section{INTRODUCTION}

One of the aims of the decoherent histories approach to quantum theory is to supply an account of the emergence of an approximately classical world from an underlying quantum one $[1,2,3,4]$. To date, most of the effort in this direction has concentrated on systems in which there is a natural separation into distinguished system and environment. In the quantum Brownian motion model, for example the distinguished system is a large particle, and the environment a bath of harmonic oscillators. Histories of imprecise positions of the distinguished system are then typically decoherent as a result of their interaction with the environment, and the probabilities for histories of position are typically peaked about classical equations of motion, with dissipation $[3,5]$. These models have the advantage of technical simplicity - a variety of known techniques, such as path integral and the influence functional $[6,7]$, may be brought to bear, and emergent classicality is readily shown. These systems have also been analysed from other perspectives, including the density matrix approach and quantum state diffusion approach, with qualitatively similar results $[8,9,10]$.

It is however, of considerable interest to investigate models of a more general class that might describe a gas or fluid, where there are certainly no distinguished point particles, and possibly even no obvious candidates for an environment to produce decoherence. In this context it has been suggested by Gell-Mann and Hartle that for a large and possibly complex system, the variables that will become classical "habitually" are the local densities (energy, momentum and number, for example), integrated over small volumes [3]. They are expected to be approximately decoherent because they are approximately conserved (since exactly conserved quantities are exactly decoherent [11]). Furthermore, there is some hope that the probabilities for such histories might be peaked about interesting deterministic evolution equations. This is because it is known that the local densities, averaged in a 
local equilibrium state, obey hydrodynamic equations.

It is therefore of interest to study the decoherent histories approach applied to systems consisting of large numbers of particles, with histories consisting of projections onto local densities, with the aim of showing, first, that these histories decohere; and second, their probabilities are peaked about hydrodynamic equations. In this paper we make a number of steps in this direction.

Generally, we are concerned with systems which are described at the microscopic level by a Hamiltonian of the form

$$
H=\sum_{i}\left(\frac{\mathbf{p}_{i}^{2}}{2 m_{i}}+\sum_{i \neq j} V_{i j}\left(\left|\mathbf{q}_{i}-\mathbf{q}_{j}\right|\right)\right)
$$

The local densities of interest are the number density $n(\mathbf{x}, t)$, the momentum density $\mathbf{g}(\mathbf{x}, t)$ and the energy density $h(\mathbf{x}, t)$, defined by,

$$
\begin{aligned}
& n(\mathbf{x}, t)=\sum_{i} \delta\left(\mathbf{x}-\mathbf{q}_{i}\right) \\
& \mathbf{g}(\mathbf{x}, t)=\sum_{i} \mathbf{p}_{i} \delta\left(\mathbf{x}-\mathbf{q}_{i}\right) \\
& h(\mathbf{x}, t)=\sum_{i}\left(\frac{\mathbf{p}_{i}^{2}}{2 m_{i}}+\sum_{j \neq i} V_{i j}\left(\left|\mathbf{q}_{i}-\mathbf{q}_{j}\right|\right)\right) \delta\left(\mathbf{x}-\mathbf{q}_{i}\right)
\end{aligned}
$$

When these objects are raised to the status of operators in the quantum theory, pairs of non-commuting operators are symmetrized to ensure hermiticity. (In what follows it will be assumed, where necessary, that non-commuting operators are ordered in this way, even though it will not always be written explicitly). The standard derivation of the hydrodynamic equations for the average values of the local densities starts by considering the continuity equations expressing local conservation [12]. These are of the form

$$
\frac{\partial \sigma}{\partial t}+\nabla \cdot \mathbf{j}=0
$$


where $\sigma$ denotes $n, \mathbf{g}$ or $h$ (and the current $\mathbf{j}$ is a second rank tensor in the case of $\mathbf{g}$ ). It is assumed that, for a wide variety of initial states, conditions of local equilibrium are established after a short period of time. This means that on scales small compared to the overall size of the fluid, but large compared to the microscopic scale, equilibrium conditions are reached in each local region, characterized by a local temperature, pressure etc. which vary slowly from region to region. This state of affairs is described by a local equilibrium density operator, which has the form

$$
\rho=Z^{-1} \exp \left(-\int d^{3} x \beta(\mathbf{x})[h(\mathbf{x})-\bar{\mu}(\mathbf{x}) n(\mathbf{x})-\mathbf{u}(\mathbf{x}) \cdot \mathbf{g}(\mathbf{x})]\right)
$$

where $\beta, \bar{\mu}$ and $\mathbf{u}$ are Lagrange multipliers and are slowly varying functions of space and time. ( $\beta$ is the inverse temperature, $\mathbf{u}$ is the average velocity field, and $\bar{\mu}$ is related to the chemical potential which in turn is related to the average number density). The hydrodynamic equations follow when the continuity equations are averaged in this state. The averaging produces averages of the currents, $\langle\mathbf{j}\rangle$, but constitutive relations then typically follow, which relate the averages of the currents to averages of the local densities (and their derivatives). Hence one obtains a closed set of evolution equations for $\langle n\rangle,\langle\mathbf{g}\rangle$ and $\langle h\rangle$ (smeared over small spatial volumes).

What is therefore known already is that the average values of the local densities, in the local equilibrium state, obey hydrodynamic equations.

Demonstrating emergent classicality, however, requires considerably more than this. The above result is not unlike the Ehrenfest theorem of elementary quantum mechanics. This shows that the expectation values of position and momentum obey classical equations of motion, but it alone does not constitute a derivation of the emergence of classical mechanics from quantum mechanics [13].

Here, we adopt the discussion of emergent classicality used in the decoherent histories 
approach. In that approach we consider the decoherence functional,

$$
D\left(\underline{\alpha}, \underline{\alpha}^{\prime}\right)=\operatorname{Tr}\left(P_{\alpha_{n}}\left(t_{n}\right) \cdots P_{\alpha_{1}}\left(t_{1}\right) \rho P_{\alpha_{1}^{\prime}}\left(t_{1}\right) \cdots P_{\alpha_{n}^{\prime}}\left(t_{n}\right)\right)
$$

The histories are characterized by projectors $P_{\alpha}(t)$ (in the Heisenberg picture) at each moment of time, in this case onto the local densities (the detailed construction is described below). The decoherent histories approach is described in detail in many places, so we will not go into detail here $[1,2,3,4,5,14]$. Briefly, the histories are said to be decoherent when the decoherence functional (1.7) is approximately zero in its off-diagonal terms. Probabilities equal to the diagonal elements may then be assigned to the histories in the set.

A decoherent histories derivation of the hydrodynamic equations goes beyond the standard one in two respects. First of all, it shows that histories of local densities are decoherent, so probabilities can be assigned to them. Secondly, rather than considering just the evolution of average values of the local densities, we consider the probability for histories of values, which is a far more detailed object. We will, however, find an important simplifying feature in the special case of a local equilibrium initial state: this is that the probabilities for histories are strongly peaked about the evolution of the average values. In this case, the jump from the evolution of average values to probabilities for histories is then in fact not that large.

It is perhaps also of interest to note that it is difficult to discuss the decoherence and emergent classicality of the local densities using other approaches to decoherence. The density matrix approach, for example, relies quite heavily on the existence of a reduced density matrix for the variables followed, e.g., the position of Brownian particle, after the variables ignored are traced out. This requires that the total Hilbert space is a tensor product of the Hilbert space of the variables followed with the Hilbert space of the en- 
vironment, which is not the case here. The decoherent histories approach, by contrast, adapts more comfortably to these situations. It deals with projections onto subspaces of the total Hilbert space, and does not require a tensor product structure.

Turning to this paper, we will address the issue of the emergence of hydrodynamic equations for the special case of the diffusion of local number density of foreign particles in a fluid. We then go on to argue that the approach and some of the results also apply to the more general case; thus, a (partially heuristic) derivation of the hydrodynamic equations from decoherent histories will be given.

The system we are initially interested in is a fluid containing a very low concentration of foreign particles. The number of foreign particles is conserved, and it is well-known that the local number density obeys the diffusion equation. For us, the simplifying feature is that the microscopic dynamics of the foreign particles is reasonably simple, and the approach to equilibrium and the consitutive relations are easily derived. In particular, each foreign particle may be regarded as a free particle in a bath (the background fluid), so is essentially a particle undergoing quantum Brownian motion, the description of which is well-known.

Hence, in this particular model there $i s$ in fact, an environment, namely the background fluid, which assists in many ways, in particular it makes a contribution to decoherence. What is new, however, compared to previously considered system-environment models, is to consider histories of local densities of many Brownian particles, rather than histories of positions of a single point particle. Indeed, this model is a kind of half-way house between the familiar system-environment models and true hydrodynamic models. Furthermore, as indicated above, the model is sufficiently similar to the proper hydrodynamic case to make its results of relevance. 
The quantum Brownian motion model and its diffusive limit is described in Section II. Its reinterpretation as a deterministic equation for the number density is described in Section III. The decoherent histories analysis is described in Section IV. It is shown that histories are peaked about diffusive evolution, and that they are approximately decoherent. The extent to which these ideas go over to the more general case is discussed in Section V. We summarize and conclude in Section VI. An alternative proof of the key results of Sections IV and V is given in an appendix.

A number of other authors have investigated closely related issues. The present work is strongly rooted in the decoherent histories approach to quantum theory of Gell-Mann and Hartle, who have frequently stressed that the most general situations in which there is decoherence and peaking of the probabilities about deterministic equations are generally not of the system-environment type $[2,3,13]$. An earlier paper investigated the decoherence of histories of number density in a simple model involving spin waves [15]. Brun and Hartle have investigated coarse grainings in an oscillator chain model which allow a wide variety of different divisions into system and environment, and also lead to a wave equation as the effective classical equation of motion [16]. Calzetta and Hu have investigated the decoherence of histories specified by fixed values of certain correlation functions in a field theory [17]. Other coarse graining schemes not of the system environment type (and not involving decoherent histories) may be found in Refs.[18,19]. Some related issues concerning the derivation of hydrodynamic equations may be found in Ref.[20]. Omnès [21] has observed that the work of Feffermann [22] may contain some useful guides concerning the selection of a preferred set of variables is a general system.

Finally, it should be mentioned that not all authors agree that the decoherent histories approach is capable of explaining the emergence of classical physics from an underlying quantum theory. The various sides of this rather subtle debate are described in 
Refs.[23,24,25]. The present paper is essentially concerned with exploring the mathematical properties of the decoherent histories approach, as it currently exists, and has little to add to this debate. The extent to which the decoherent histories approach remains a valuable and useful one is perhaps best summarized in Ref.[26].

\section{DERIVATION OF THE DIFFUSION EQUATION}

The diffusion equation arises for a system consisting of a collection of $N$ foreign particles in a fluid of a much larger number of background particles. We assume that the density of foreign particles is so low that their interactions with each other may be neglected. Each particle interacts only with the fluid which we assume is in equilibrium at temperature $T$. Each individual foreign particle, therefore, may be modeled as a free Brownian particle for which the fluid plays the role of a thermal environment. The description of this system is well known. Classically, the phase space density $W(p, q)$ of the particle obeys a FokkerPlanck equation of the Kramers type, which takes the form [27],

$$
\frac{\partial W}{\partial t}=-\frac{p}{M} \frac{\partial W}{\partial q}+2 \gamma \frac{\partial}{\partial p}(p W)+2 M \gamma k T \frac{\partial^{2} W}{\partial p^{2}}
$$

In the quantum theory, the Wigner function obeys the same equation (in the high temperature limit, which we work in here), The density operator is recovered from the Wigner function by the transformation

$$
\rho(x, y)=\int d p e^{\frac{i}{\hbar} p(x-y)} W\left(p, \frac{x+y}{2}\right)
$$

and it obeys the master equation

$$
\begin{aligned}
\frac{\partial \rho}{\partial t}= & \frac{i \hbar}{2 M}\left(\frac{\partial^{2} \rho}{\partial x^{2}}-\frac{\partial^{2} \rho}{\partial y^{2}}\right) \\
& -\gamma(x-y)\left(\frac{\partial \rho}{\partial x}-\frac{\partial \rho}{\partial y}\right)-\frac{2 M \gamma k T}{\hbar^{2}}(x-y)^{2} \rho
\end{aligned}
$$


This equation arises directly in the Caldeira-Leggett model of quantum Brownian motion, in which a particle is linearly coupled to a thermal bath of harmonic oscillators [7]. However, there are many indications $[28,29]$ that the form of this equation is in fact a lot more general, and will still hold, in some approximation, for more complicated and realistic couplings.

Eq.(2.1) may be solved by introducing the propagator $K\left(p, q, t \mid p_{0}, q_{0}, 0\right)$, defined by

$$
W(p, q, t)=\int d p_{0} d q_{0} K\left(p, q, t \mid p_{0}, q_{0}, 0\right) W_{0}(p, q)
$$

The propagator also obeys Eq.(2.1) with the initial conditions,

$$
K\left(p, q, 0 \mid p_{0}, q_{0}, 0\right)=\delta\left(p-p_{0}\right) \delta\left(q-q_{0}\right)
$$

The explicit form is,

$$
K\left(p, q, t \mid p_{0}, q_{0}, 0\right)=\exp \left(-\alpha\left(p-p^{c l}\right)^{2}-\beta\left(q-q^{c l}\right)^{2}-\epsilon\left(p-p^{c l}\right)\left(q-q^{c l}\right)\right)
$$

(ignoring prefactors, which may be recovered by normalization where necessary). Here,

$$
\begin{aligned}
& q^{c l}=q_{0}+\frac{p_{0}}{2 M \gamma}\left(1-e^{-2 \gamma t}\right) \\
& p^{c l}=p_{0} e^{-2 \gamma t}
\end{aligned}
$$

Explicit expressions for the coefficients $\alpha, \beta, \epsilon$ may be found in Ref.[30].

We are interested in the behaviour of this system for times $t>>\gamma^{-1}$. Unlike the harmonic oscillator, this does not settle down to equilibrium, but certain simplifications do occur. First of all, note that

$$
\begin{aligned}
& q^{c l} \rightarrow q_{0}+\frac{p_{0}}{2 M \gamma} \\
& p^{c l} \rightarrow 0
\end{aligned}
$$


Second, from the explicit expressions in Ref.[30], it can be shown that

$$
\alpha \rightarrow \frac{1}{2 M k t}, \quad \beta \rightarrow \frac{M \gamma}{2 k T t}, \quad \epsilon \rightarrow-\frac{1}{2 k T t}
$$

The solution Eq.(2.1) therefore approaches the form,

$$
W(p, q, t)=\int d p_{0} d q_{0} \exp \left(-\alpha p^{2}-\beta\left(q-q^{c l}\right)^{2}-\epsilon p\left(q-q^{c l}\right)\right) W_{0}(p, q)
$$

The distribution of momenta is

$$
g(p)=\int d q W(p, q, t) \approx \exp \left(-\frac{p^{2}}{2 M k T}\right)
$$

(where we have used $\gamma t>>1$ ). Hence the momentum distribution approaches the MaxwellBoltzmann form. The distribution of positions, on the other hand, approaches the form

$$
\begin{aligned}
f(q, t) & =\int d p W(p, q, t) \\
& \approx \int d p_{0} d q_{0} \exp \left(-\frac{M \gamma}{2 K T t}\left(q-q_{0}-\frac{p_{0}}{2 M \gamma}\right)^{2}\right) W_{0}\left(p_{0}, q_{0}\right)
\end{aligned}
$$

It follows that $f(q, t)$ obeys the diffusion equation,

$$
\frac{\partial f}{\partial t}=D \frac{\partial^{2} f}{\partial q^{2}}
$$

where $D=k T / 2 M \gamma$.

This equation can also be derived by integrating Eq.(2.1) over $p$, with the result,

$$
\frac{\partial f}{\partial t}=-\frac{1}{M} \frac{\partial}{\partial q} \int d p p W(p, q, t)
$$

and Eq.(2.10) may be then be used to show that,

$$
\int d p p W(p, q, t)=-\frac{k T}{2 \gamma} \frac{\partial}{\partial q} f(q)
$$

hence the diffusion equation follows. 


\section{DIFFUSION OF NUMBER DENSITY}

Turn now from the case of a single particle to the system of interest - a collection of $N$ particles, each one of which is described by the one-particle dynamics outlined above. The number density at the point $x$ is

$$
n(x)=\sum_{k=1}^{N} \delta\left(q_{k}-x\right)
$$

Usually, we are interested in the number density smeared over a small volume $V$, which we write,

$$
n_{V}(x)=\sum_{k=1}^{N} \delta_{V}\left(q_{k}-x\right)
$$

where $\delta_{V}$ denotes a delta function smeared over $V$, i.e., a window function equal to 1 in an interval of size $V$ and 0 outside, and $x$ will then be a discrete label. (We will not explicitly use the smeared form unless necessary). Microscopically, we assume that the $N$ particle system is described by the phase space distribution function

$$
\mu\left(p_{1}, p_{2}, \cdots p_{N}, q_{1}, q_{2}, \cdots q_{N}\right)=\prod_{k=1}^{N} W\left(p_{k}, q_{k}, t\right)
$$

where $W(p, q, t)$ are the one-particle Wigner functions of the previous section. The product form is reasonable since the particles do not interact, and even if they are initially correlated, these correlation will eventually be lost to the environment of each particle.

The number density $n(x)$ is a function of the stochastic variables $q_{k}$. However, we will show that the evolution of $n(x)$ is in fact strongly peaked about deterministic evolution according to the diffusion equation. This is, of course, almost obvious, since all we are doing is in essence reinterpreting the one-particle probability as a distribution function on a real ensemble of $N$ particles. But for what follows, it is worth going through the steps. 
First we show that the average value obeys the diffusion equation, then we show that the distribution is strongly peaked about the average.

Consider first the average of $n(x)$ in the $N$ particle distribution (3.3). It is,

$$
\begin{aligned}
\langle n(x)\rangle & =\sum_{k}\left\langle\delta\left(q_{k}-x\right)\right\rangle_{N} \\
& =N\langle\delta(q-x)\rangle_{1} \\
& =N \int d p W(p, x, t)=N f(x)
\end{aligned}
$$

where $\langle\cdots\rangle_{N}$ and $\langle\cdots\rangle_{1}$ denote averages in the $N$-particle and one-particle distributions, respectively. Hence $\langle n(x)\rangle$ obeys the diffusion equation, since $f(x)$ does, as shown in the previous section.

It is, however, enlightening to briefly demonstrate this a different way, more closely related to standard derivations of hydrodynamics equations. The number density obeys the local conservation law,

$$
\frac{\partial n}{\partial t}+\frac{1}{M} \frac{\partial g}{\partial x}=0
$$

where

$$
g(x)=\sum_{k} p_{k} \delta\left(q_{k}-x\right)
$$

Averaging through (3.5), we find we need to compute

$$
\begin{aligned}
\langle g(x)\rangle & =\sum_{k}\left\langle p_{k} \delta\left(q_{k}-x\right)\right\rangle_{N} \\
& =N \int d p p W(p, q, t)
\end{aligned}
$$

We have already computed this in Eq.(2.15). Hence we derive the constitutive relation,

$$
\langle g(x)\rangle=-\frac{k T}{2 \gamma} \frac{\partial}{\partial x}\langle n(x)\rangle
$$


and the diffusion equation for $\langle n(x)\rangle$ follows. Note also that the state averaged in here is close to a local equilibrium state - the momenta are in a Maxwell-Boltzmann distribution (2.11), the temperature $T$ is constant throughout the system, the average velocity flow is zero, and only the number density varies throughout the system (although the correlation between $p$ and $q$ in (2.10) is important for (3.8) to be obtained).

Now consider the variance of $n(x)$ in the distribution (3.3). Here we have to consider the smeared from (3.2). We have

$$
\left\langle n_{V}\right\rangle=\sum_{k}\left\langle\delta_{V}\left(q_{k}-x\right)\right\rangle_{N}=N\left\langle\delta_{V}(q-x)\right\rangle_{1}
$$

and

$$
\begin{aligned}
\left\langle n_{V}^{2}\right\rangle & =\sum_{j k}\left\langle\delta_{V}\left(q_{j}-x\right) \delta_{V}\left(q_{k}-x\right)\right\rangle_{N} \\
& =\sum_{j}\left\langle\delta_{V}^{2}\left(q_{j}-x\right)\right\rangle_{N}+\sum_{j \neq k}\left\langle\delta_{V}\left(q_{j}-x\right)\right\rangle_{N}\left\langle\delta_{V}\left(q_{k}-x\right)\right\rangle_{N} \\
& =N\left\langle\delta_{V}^{2}(q-x)\right\rangle_{1}+\left(N^{2}-N\right)\left\langle\delta_{V}(q-x)\right\rangle_{1}^{2}
\end{aligned}
$$

Therefore,

$$
\frac{\left\langle n_{V}^{2}\right\rangle-\left\langle n_{V}\right\rangle^{2}}{\left\langle n_{V}\right\rangle^{2}}=\frac{1}{N} \frac{\left\langle\delta_{V}^{2}\right\rangle-\left\langle\delta_{V}\right\rangle^{2}}{\left\langle\delta_{V}\right\rangle^{2}}
$$

For large $N$, which we assume, the distribution of $n_{V}(x)$ is very strongly peaked about its mean value.

The above result, which is a form of the central limit theorem, is important in the derivation of more general hydrodynamic equations (and indeed is used again in the next section in a slightly different form). It essentially relies on two features of the macroscopic system. Firstly, that the microscopic distrbution is of the form (3.3), i.e., a product of identical one-particle distribution functions. Secondly, that the macroscopic variable in question, such as (3.1), is a sum of identical terms each of which involves only the 
one-particle variables. It is, however, very likely (and probably provable) that the result will hold more generally. In particular, we expect it to continue to hold in the more realistic situation where the microscopic distribution function involves a certain amount of correlation between particles (so is not exactly of the product form) and when the macroscopic variables involve interaction terms between particles. Generally, we expect strong peaking about the mean values of a macroscopic variable when fixed values of that variable correspond to a very large number of similar microstates.

\section{HISTORIES OF NUMBER DENSITIES}

\section{4(A). Probabilities and Decoherence}

Consider now the question of histories of number densities. After tracing out the environment for each of the $N$ particles, the decoherence functional for histories characterized by projections onto number density at two moments of time is

$$
D\left(\bar{n}_{1}, \bar{n}_{2} \mid \bar{n}_{1}^{\prime}, \bar{n}_{2}\right)=\operatorname{Tr}\left(P_{\bar{n}_{2}} K_{0}^{t}\left[P_{\bar{n}_{1}} \rho P_{\bar{n}_{1}^{\prime}}\right]\right)
$$

The trace is over the Hilbert space of the $N$ particles. $K_{0}^{t}$ is a product of $N$ reduced density matrix propagators (corresponding to the master equation (2.3)), one for each Brownian particle. $P_{\bar{n}}$ denotes a projector onto number density, and may be represented as

$$
P_{\bar{n}(x)}=\delta_{\sigma}[\hat{n}(x)-\bar{n}(x)]
$$

Here, $\delta_{\sigma}$ is a delta function smeared over a range $\sigma$. The number density operator $\hat{n}(x)$ may be smeared over a range $V$ when necessary. (More detailed representations of projections 
onto number density are possible, in terms of projections onto position of the individual particls [15], but this is not necessary here). Eq.(4.1) is the decoherence functional for the simplest non-trivial case of two-times histories, but the arguments present below will also go through for histories of projectors at many moments of time.

For the initial state $\rho$, let us again suppose that it is a product of $N$ identical terms, as in the previous section. We will also assume that it has already evolved for a time $t>>\gamma^{-1}$, so we are in the diffusive regime. We will consider later the consequences of other types of initial state.

If the initial density matrix is a product of $N$ identical terms, we expect a central limit theorem result to hold, and the distribution of $n(x)$ in the initial state will be strongly peaked about its mean. In more detail, suppose we have a pure initial state on the $N$ particle Hilbert space which is a product of $N$ identical terms,

$$
|\Psi\rangle=|\psi\rangle \otimes|\psi\rangle \otimes \cdots \otimes|\psi\rangle
$$

Then for large $N,|\Psi\rangle$ is approximately an eigenstate of the number density operator (2.1),

$$
\hat{n}(x)|\Psi\rangle \approx\langle n(x)\rangle|\Psi\rangle
$$

where $\langle n(x)\rangle=N|\psi(x)|^{2}$. This is essentially the Finkelstein-Graham-Hartle theorem [31], since the number density is the same as the relative frequency operator (up to an overall factor of $N$ ). For projections onto number density a similar result follows, that is,

$$
\delta_{\sigma}[\hat{n}(x)-\bar{n}(x)]|\Psi\rangle \approx \delta_{\sigma}[\langle n(x)\rangle-\bar{n}(x)]|\Psi\rangle
$$

It is straightforward to generalise to the case of mixed states. That is, for a density matrix

$$
\rho=\rho_{1} \otimes \rho_{1} \otimes \cdots \otimes \rho_{1}
$$


it may be shown that

$$
\hat{n}(x) \rho \approx\langle n(x)\rangle \rho
$$

and thus, the analogue of (4.5) holds.

These results mean two things. First, that

$$
P_{\bar{n}_{1}} \rho P_{\bar{n}_{1}^{\prime}} \approx 0, \quad \text { for } \quad \bar{n}_{1} \neq \bar{n}_{1}^{\prime}
$$

Hence, there is approximate decoherence for initial states of this type. Secondly, they allow us to compute the probabilities. We have

$$
P_{\bar{n}_{1}} \rho P_{\bar{n}_{1}} \approx \delta_{\sigma}\left[\langle\hat{n}(x)\rangle-\bar{n}_{1}(x)\right] \rho
$$

Inserting into the diagonal elements of the decoherence functional (4.1), we find

$$
p\left(\bar{n}_{2}, \bar{n}_{1}\right)=\operatorname{Tr}\left(P_{\bar{n}_{2}} \rho_{t}\right) \quad \delta_{\sigma}\left[\langle\hat{n}(x)\rangle-\bar{n}_{1}(x)\right]
$$

Now $\rho_{t}$ is the evolution of the initial state under the non-unitary propagator $K_{0}^{t}$. The key point is that it remains in the product form, so,

$$
\operatorname{Tr}\left(P_{\bar{n}_{2}} \rho_{t}\right) \approx \delta_{\sigma}\left[\langle\hat{n}(x)\rangle_{t}-\bar{n}_{2}(x)\right]
$$

Hence, the probability (4.10) is strongly peaked about $\bar{n}_{1}(x)=\langle\hat{n}(x)\rangle$ and $\bar{n}_{2}(x)=\langle\hat{n}(x)\rangle_{t}$, that is, about evolution according to the diffusion equation.

We have therefore proved the desired result: for initial states of the form (4.6), histories of number density are decoherent, and their probabilities are peaked about diffusive evolution.

\section{4(B). More about Decoherence}

Let us consider now in more detail the question of decoherence. Since each individual foreign particle is coupled to its surrounding fluid environment, the possibility exists that 
this environment causes decoherence of each particle's position. Decoherence of number density then follows trivially, since projections onto number density may be constructed from coarse-grainings of projections onto position [15]. However, decoherence of a particle coupled to an environment requires certain choices of parameters for the model, such as large mass and large temperature. Hence, there will be certain parameter ranges (in particular, small masses for the foreign particles) for which the decoherence at the one particle level may not be very good. In this case, it is the large $N$ effect which produces the dominant contribution to decoherence of number density. (Generally, replicating the system $N$ times enhances the degree of decoherence exponentially in $N[15])$.

Because decoherence is not necessarily produced by the environment of each particle, it is of interest to see how decoherence comes about for initial states consisting of superpositions of number density. So let,

$$
\begin{aligned}
|\Psi\rangle=\frac{1}{\sqrt{2}}(|\psi\rangle & \otimes|\psi\rangle \otimes \cdots \otimes|\psi\rangle \\
& +|\chi\rangle \otimes|\chi\rangle \otimes \cdots \otimes|\chi\rangle)
\end{aligned}
$$

It is now the case that $P_{\bar{n}_{1}} \rho P_{\bar{n}_{1}^{\prime}} \neq 0$ if we take $\bar{n}_{1}(x)=N|\psi(x)|^{2}$ and $\bar{n}_{1}^{\prime}(x)=N|\chi(x)|^{2}$.

In this case, decoherence may be demonstrated by appealing to approximate determinism: decoherence follows if the probabilities are strongly peaked about a unique relationship between alternatives at different times. To see this, note that for histories consisting of alternatives at two moments of time, the decoherence functional satisfies [5]

$$
\left|D\left(\bar{n}_{1}, \bar{n}_{2} \mid \bar{n}_{1}^{\prime}, \bar{n}_{2}\right)\right|^{2} \leq p\left(\bar{n}_{1}, \bar{n}_{2}\right) p\left(\bar{n}_{1}^{\prime}, \bar{n}_{2}\right)
$$

So if $p\left(\bar{n}_{1}, \bar{n}_{2}\right)$ is strongly peaked about an equation linking $\bar{n}_{1}$ and $\bar{n}_{2}$, only one of $p\left(\bar{n}_{1}, \bar{n}_{2}\right)$ and $p\left(\bar{n}_{1}^{\prime}, \bar{n}_{2}\right)$ can be non-zero for $\bar{n}_{1} \neq \bar{n}_{1}^{\prime}$, hence the decoherence functional is approximately diagonal. 
Before looking in more detail at how this argument applies to the specific case of diffusive evolution, some qualifications must be added. For diffusive evolution there is not, in fact, a unique initial density corresponding to a fixed final one. There will generally be a spread of initial densities that evolve into a given final density, and the non-uniqueness will get worse with increasing time intervals. A fixed final density will fix the initial density precisely only for reasonably short times, and for sufficiently large choices of the width $\sigma$ of the projections onto number density (which ensures that different values of $n(x)$ are sufficiently "far apart"). Fortunately, these restrictions do not adversely affect the main objective here - to demonstrate the emergence of the diffusion equation - since it is clearly sufficient to consider projectors onto number density at two closely separated times.

Returning to the demonstration of decoherence, the probabilities $p\left(\bar{n}_{1}, \bar{n}_{2}\right)$ are non-zero only if $\bar{n}_{1}$ is equal to either $N|\psi(x)|^{2}$ or $N|\chi(x)|^{2}$. The first choice projects the initial state onto $|\psi\rangle \otimes|\psi\rangle \otimes \cdots$ and the second onto $|\chi\rangle \otimes|\chi\rangle \otimes \cdots$. The point is now that each of these two states, on evolution for a short time $t$, are strongly peaked about two distinct values of number density, as determined via Eq.(2.12). (It is not necessarily diffusive evolution,

except for times $t>>\gamma^{-1}$. It is only necessary that the final value of number density be uniquely determined by the initial state). It follows that, modulo the above remarks, $p\left(\bar{n}_{1}, \bar{n}_{2}\right)$ is strongly peaked about a unique relation between $\bar{n}_{1}$ and $\bar{n}_{2}$, hence there is approximate decoherence.

\section{4(C). Other Variables}

It is possible to consider histories of other types of variables in this diffusive model. Momentum and energy are not so interesting because they are not locally conserved - the momentum and energy of the foreign particles is exchanged with the environment. But 
another finer-grained variable that is likely to be of more general use is the phase space density. Classically, this is given by

$$
f(x, k)=\sum_{j} \delta\left(p_{j}-k\right) \delta\left(q_{j}-x\right)
$$

This quantity may be smeared over ranges of $p$ and $q$, so that it becomes a function $f_{\Gamma}(x, k)$ which counts the number of particles in the phase space cell of size $\Gamma$ labeled by discrete lables $x, k$. Classically,

$$
\langle f(x, k)\rangle_{N}=N W(k, x)
$$

where $W(k, x)$ is the one-particle phase space distribution function. Like the number

density, the distribution of $f(x, k)$ is strongly peaked about its mean, (4.15), since (4.14) is a sum of identical terms. Hence, we can regard the phase space density (4.14) as a hydrodynamic variable whose evolution equation is the Fokker-Planck equation (2.1).

Similar results will hold in the quantum theory. Operator ordering is necessary to make (4.14) hermitian. We can the consider projections onto ranges of its values,

$$
\delta_{\sigma}\left[\hat{f}_{\Gamma}(x, k)-\bar{f}(x, k)\right]
$$

The subsequent treatment will be similar to the number density case. One would expect the quantum theory to exhibit an approximate determinism in the evolution of (4.14) (similar to Omnès theorems on the evolution of phase space projectors [4]) and this will guarantee approximate decoherence of histories of phase space density. The probabilities for these histories will then be peaked about evolution according to the Fokker-Planck equation. (This will be investigated in more detail elsewhere).

\section{General Hydrodynamic Histories}

Interestingly, many of the features of the diffusive model go over to the general case. 
Consider a closed system consisting of $N$ weakly interacting particles, now without a background fluid. The number, momentum, and energy density are given by

$$
\begin{aligned}
& n(x)=\sum_{j} \delta\left(q_{j}-x\right) \\
& g(x)=\sum_{j} \delta\left(q_{j}-x\right) p_{j} \\
& h(x)=\sum_{j} \delta\left(q_{j}-x\right) \frac{p_{j}^{2}}{2 m}
\end{aligned}
$$

They are written for the case of a one-dimensional model for notational simplicity, although the three-dimensional case is readily obtained. The Hamiltonian is for a system of approximately non-interacting particles. This is sufficient to cover, for example, the case of a dilute gas. The neglect of interactions might seem restrictive, but, as in the diffusive model, we find that the interesting things we are able to say about the probabilities histories arise from large $N$ statistics, rather than from detailed knowledge of the microscopic interactions.

As in the previous case, the densities need to be smeared over a small volume. Also, in the quantum case, non-commuting operators should be ordered in such a way that they are hermitian.

\section{5(A). Decoherence and Probabilities for a Local Equilibrium Initial State}

The usual derivation of the hydrodynamic equations [12] involves assuming a local equilibrium state, which for this one-dimensional case has the form

$$
\rho=Z^{-1} \exp \left(-\int d x \beta(x)[\hat{h}(x)-\bar{\mu}(x) \hat{n}(x)-u(x) \hat{g}(x)]\right)
$$


Because of the delta-functions in (5.1)-(5.3) this becomes,

$$
\rho=Z^{-1} \exp \left(-\sum_{j} \beta\left(q_{j}\right)\left[\frac{p_{j}^{2}}{2 m}-\bar{\mu}\left(q_{j}\right)-u\left(q_{j}\right) p_{j}\right]\right)
$$

The usual manipulations on this object may be performed by assuming that the Lagrange multipliers $\beta, \bar{\mu}, u$ are slowly varying functions. In particular, one may show that the corresponding one-particle Wigner function is

$$
W_{1}(p, q)=f(q) \exp \left(-\frac{(p-m u)^{2}}{2 m k T(q)}\right)
$$

where $k T=1 / \beta$. The quantities $f(q), u$ and $T$ are assumed to be slowly varying functions of $q$ and $t$. (The quantity $f(q)$ is proportional to $e^{\beta \mu}$, where $\mu=\bar{\mu}-\frac{1}{2} m u^{2}$ ). It is straightforwardly shown that the averages in this state, $\langle n(x)\rangle,\langle g(x)\rangle,\langle h(x)\rangle$ obey a simple set of hydrodynamic equations.

In the quantum theory, projectors onto local densities are again constructed using deltafunctions. The projection onto number density is as before Eq.(4.2) and the projectors onto momentum and energy density are

$$
\begin{aligned}
& P_{\bar{g}}=\delta_{\sigma_{g}}[\hat{g}(x)-\bar{g}(x)] \\
& P_{\bar{h}}=\delta_{\sigma_{h}}[\hat{h}(x)-\bar{h}(x)]
\end{aligned}
$$

Again $\delta_{\sigma}$ denotes a delta function smeared over a range $\sigma$. Strictly, there is one such delta-function for each value of $x$ (which is discrete if $\delta\left(q_{j}-x\right)$ is smeared over a small range). Although the operators $\hat{n}(x), \hat{g}(x), \hat{h}(x)$ do not commute, the projections onto ranges of their values $P_{\bar{n}}, P_{\bar{g}}, P_{\bar{h}}$ will approximately commute with each other if both the width $\sigma$ of the projections and the smearing volume of $\delta\left(q_{j}-x\right)$ is sufficiently large. (The extent to which this approximation is good will also depend to some extent on the initial state they are traced with in the decoherence functional). 
In the approximation that these projectors commute, the decoherence functional for a two time hydrodynamic history are

$$
D\left(\alpha_{1}, \alpha_{2} \mid \alpha^{\prime}, \alpha_{2}\right)=\operatorname{Tr}\left(P_{\bar{n}_{2}} P_{\bar{g}_{2}} P_{\bar{h}_{2}} e^{-i H t} P_{\bar{n}_{1}} P_{\bar{g}_{1}} P_{\bar{h}_{1}} \rho P_{\bar{n}_{1}} P_{\bar{g}_{1}} P_{\bar{h}_{1}} e^{i H t}\right)
$$

We take the initial state $\rho$ to be the local equilibrium state (5.4). From what has gone before, it should be clear that results similar to (4.8), (4.9) in the diffusive model hold. That is, if the widths of the projections $\sigma_{n}, \sigma_{g}, \sigma_{h}$ are much greater than the variances of the corresponding operators in the local equilibrium state, we have

$$
P_{\bar{n}_{1}} P_{\bar{g}_{1}} P_{\bar{h}_{1}} \rho P_{\bar{n}_{1}^{\prime}} P_{\bar{g}_{1}^{\prime}} P_{\bar{h}_{1}^{\prime}} \approx 0
$$

for $\bar{n}_{1} \neq \bar{n}_{1}^{\prime}, \bar{g}_{1} \neq \bar{g}_{1}^{\prime}, \bar{h}_{1} \neq \bar{h}_{1}^{\prime}$. Hence there is decoherence for this initial state. Similarly,

$$
\begin{aligned}
\bar{P}_{\bar{n}_{1}} P_{\bar{g}_{1}} P_{\bar{h}_{1}} \rho P_{\bar{n}_{1}} P_{\bar{g}_{1}} P_{\bar{h}_{1}} \approx & \delta_{\sigma_{n}}\left[\langle\hat{n}(x)\rangle-\bar{n}_{1}(x)\right] \\
& \times \delta_{\sigma_{g}}\left[\langle\hat{g}(x)\rangle-\bar{g}_{1}(x)\right] \delta_{\sigma_{h}}\left[\langle\hat{h}(x)\rangle-\bar{h}_{1}(x)\right] \rho
\end{aligned}
$$

These two results follow from the fact that the local equilibrium state is very strongly peaked in the locally conserved quantities about their average values for large $N$. The proof of this property of the equilibrium state is similar to the proof, in Section 3 , that the distribution of number density in the diffusive model is strongly peaked about its mean. That proof relied on the property that the averages of $n_{V}$ and $n_{V}^{2}$ are sums of identical terms involving averages in the one-particle distribution functions (see Eqs.(3.9)(3.11)). In the local equilibrium state, the averages of functions of the local densities are not exactly sums of identical terms, since the parameters of the distribution, such as $\beta$, are not constant. That is, the one-particle distribution functions such as (5.6) are not quite the same from one particle to another. The required peaking about the mean will still hold, however, because the parameters of the local equilibrium state are slowly varying functions. 
After being hit by the initial projection, the density matrix is evolved to time $t$, where it is still a local equilibrium state, and hitting it again with a projection yields the average values at time $t$. Hence,

$$
\begin{aligned}
p\left(\alpha_{1}, \alpha_{2}\right) & \approx \delta_{\sigma_{n}}\left[\langle\hat{n}(x)\rangle_{t}-\bar{n}_{2}(x)\right] \delta_{\sigma_{g}}\left[\langle\hat{g}(x)\rangle_{t}-\bar{g}_{2}(x)\right] \delta_{\sigma_{h}}\left[\langle\hat{h}(x)\rangle_{t}-\bar{h}_{2}(x)\right] \\
& \times \delta_{\sigma_{n}}\left[\langle\hat{n}(x)\rangle-\bar{n}_{1}(x)\right] \delta_{\sigma_{g}}\left[\langle\hat{g}(x)\rangle-\bar{g}_{1}(x)\right] \delta_{\sigma_{h}}\left[\langle\hat{h}(x)\rangle-\bar{h}_{1}(x)\right]
\end{aligned}
$$

The probability distribution is therefore peaked about the hydrodynamic equations. (An alternative derivation of this result, using Gaussian projections, is given in Appendix A).

This is the main result of this section: for a local equilibrium initial condition, histories of local densities are approximately decoherent and their probabilities are peaked about evolution according to the hydrodynamic equations.

The discussion of finer-grained histories involving projections onto phase space density, Eqs.(4.14)-(4.16) also applies to the more general case. Here, the phase space density is not unlike a locally conserved quantity, since it can change only as a result of a net flow in phase space over the boundary of the local phase space cell. (This is not the case in the diffusive model). The main difference is that in the general case we would expect the oneparticle phase space density to obey a Boltzmann equation, rather than a Fokker-Planck equation (2.1).

\section{5(B). Decoherence More Generally}

Turn now to the question of decoherence for more general initial states. As stated in the Introduction, we expect the approximate conservation of the local densities to ensure their approximate decoherence. This is because exactly conserved quantities are exactly 
decoherent [11], which may be seen as follows. Consider the two-time decoherence functional:

$$
D\left(\alpha_{1}, \alpha_{2}, \mid \alpha_{1}^{\prime}, \alpha_{2}\right)=\operatorname{Tr}\left(P_{\alpha_{2}} e^{-i H t} P_{\alpha_{1}} \rho P_{\alpha_{1}^{\prime}} e^{i H t}\right)
$$

Suppose the projections are onto some exactly conserved quantity. Then $P_{\alpha_{2}}$ commutes with $H$, so it may be moved through the unitary evolution operator to act directly on $P_{\alpha_{1}}$ or $P_{\alpha_{1}^{\prime}}$. Since $P_{\alpha_{2}} P_{\alpha_{1}}=0$ unless $\alpha_{2}=\alpha_{1}$, we see that the decoherence functional is zero unless $\alpha_{1}=\alpha_{2}=\alpha_{1}^{\prime}$, hence there is decoherence, for every initial state.

More generally, there will also be (approximate) decoherence if the quantum theory exhibits some kind of approximate determinism. Suppose that evolution of the projectors is such that $e^{i H t} P_{\alpha_{2}} e^{-i H t} \approx P_{\alpha_{2}(t)}$. That is, it is approximately equal to another projector of the same operator (but onto a different range, labeled by $\alpha_{2}(t)$ ). Then again the decoherence functional is approximately zero unless $\alpha_{1}=\alpha_{2}(t)=\alpha_{1}^{\prime}$. An example of this type of decoherence may be found in the histories of phase space projectors, considered by Omnès. He showed that a projector onto a sufficiently large cell $\Gamma_{0}$ of phase space evolves under unitary evolution into another phase space projector onto the phase space cell $\Gamma_{t}$, the classical evolution of $\Gamma_{0}$.

In a similar way, we can argue for the decoherence of local densities for a wide class of initial states by considering the time evolution of the final projector. Take it to be of the form

$$
P_{\alpha_{2}}=\delta_{\sigma_{n}}\left[\hat{n}(x)-\bar{n}_{2}(x)\right] \delta_{\sigma_{g}}\left[\hat{g}(x)-\bar{g}_{2}(x)\right] \delta_{\sigma_{h}}\left[\hat{h}(x)-\bar{h}_{2}(x)\right]
$$

and similarly for the initial projectors, $P_{\alpha_{1}}$. The question of decoherence is the question of the general form of the object,

$$
P_{\alpha_{2}}(t)=e^{i H t} P_{\alpha_{2}} e^{-i H t}
$$


In particular, if this object is strongly localized in $n, g$ and $h$, then there will be approximate decoherence, since

$$
P_{\alpha_{1}^{\prime}} P_{\alpha_{2}}(t) P_{\alpha_{1}} \approx 0, \quad \text { for } \quad \alpha_{1} \neq \alpha_{1}^{\prime}
$$

This is of course an operator statement, so involves some kind of restriction on the class of initial states $\rho$ it is traced with in the decoherence functional. But we would expect it to be true for a wide class of states, although clearly not all.

To see why (5.16) should hold, regard the final projector as representing a final state, $\rho_{f}=P_{\alpha_{2}} / \operatorname{Tr}\left(P_{\alpha_{2}}\right)$ (the trace is finite). So Eq.(5.15) is the backwards evolution of this state,

$$
\rho_{f}(-t)=e^{i H t} \rho_{f} e^{-i H t}
$$

Now, for unitary evolution, evolution backwards in time has the same generic physical features as evolution forwards in time. In particular, it is strongly believed that a wide class of initial conditions for a large many particle system will quickly evolve to a local equilibrium state [32]. Therefore, we expect that for sufficiently large $t(5.17)$ will be close to a local equilibrium state, hence will be localized in $n, g$ and $h$, and so (5.16) will hold.

The issue is further helped along by the observation that $P_{\alpha_{2}}$ and hence $\rho_{f}$ is initially localized in $n, g$ and $h$, and, since these are locally conserved quantities, both they and their fluctuations will vary slowly. In fact, $\rho_{f}$ is closely related to a local equilibrium state. It is in a sense the microcanonical version of it. In $\rho_{f}$, the variables $n, g$ and $h$ are constrained to lie in a small range centred about $\bar{n}, \bar{g}$ and $\bar{h}$. In the local equilibrium state, on the other hand, only the averages of $n, g$ and $h$ are constrained to take set values, but large $N$ statistics guarantees that the fluctuations about the mean are extremely small, hence the distributions of $n, g$ and $h$ are here also concentrated in a small range centred about the mean. 
Since $\rho_{f}$ is either essentially equivalent to a local equilibrium state, or very close to one, and since a local equilibrium state remains so under unitary evolution, it is very plausible that (5.17) is localized in the hydrodynamic variables (and in particular it is probably a local equilibrium state), so (5.16) will hold. We therefore expect decoherence for a wide variety of initial states.

It is perhaps pertinent to elaborate a little on what it means for a state to approach the local equilibrium form (5.4). We expect, for instance, a physical system starting out in a pure initial state to approach local equilibrium, in some sense. Yet (5.4) is a very mixed state so cannot be reached from a pure state by unitary evolution. What is going on here is that one is typically interested only in the averages of the local densities, and for these purposes, the quantum state of the system (which may be pure) can be replaced with another state possessing the same average values. Usually, the replacement state is chosen by the maximum entropy method (subject to fixed average values), and it is this which leads to the local equilibrium state (5.4) [33].

Differently viewed, since the local densities are sums of one-particle operators, their averages involve only the one-particle distributions functions, such as (5.6). (If interactions are significant two-particle operators and distribution functions could be involved). These distribution functions may exhibit the local equilibrium form (5.6), even though the actual state of the whole system is not of this type. The local equilibrium density operator of the whole system, (5.4), is the effective state of the whole system given that the one-particle distribution functions are of the form (5.6).

In this connection, it is also worth mentioning a different argument for decoherence. We are interested in histories of projections onto number, momentum and energy density on the system of $N$ particles. However, as indicated in Ref.[15], these projectors may be expressed in terms of a (generally rather complicated) sum of projections involving 
each individual particle. Hence, we could compute the decoherence functional for the local densities by first considering the decoherence functional for the much finer-grained histories involving the position, momentum density and energy density of each particle. Now the point is that the decoherence functional for each individual particle would then have the familiar system-environment form, in which the properties of one or two particles are followed and all the rest are traced out. On general grounds, we would therefore expect a degree of decoherence of the properties of each individual particle. Since the local densities of the $N$ particle system are constructed from coarse grainings of the individual particle histories, the degree of decoherence can only improve (as seen explicitly in Ref.[15]). From this point of view, the hydrodynamic models are in fact very similar to the diffusive model of this paper, except that here each particle doubles up as both system and environment.

The main difference, however, between the hydrodynamic models and the usual systemenvironment models, is that there are no distinguished particles, since they will typically all have the same mass. So although there may be a degree of decoherence in the one-particle reduced density matrices, unlike the quantum Brownian motion models, there will be little determinism in the evolution of the single particle positions and momenta, because the fluctuations will be very large as a result of interacting with an "environment" consisting of large numbers of particle of the same mass as the "system". This, in fact, is one of the reasons it is interesting of consider instead histories of local densities of the $N$ particle system - averaging over very large numbers of particles smooths out the fluctuations, so that the local densities evolve almost deterministically. 


\section{SUMMARY AND CONCLUSIONS}

For a local equilibrium initial state, the derivation of hydrodynamic equations from decoherent histories is reasonably simple. Large $N$ statistics guarantees that the probabilities for histories of local densities are strongly peaked about a single history - the evolution of the mean in the local equilibrium initial state. We therefore readily connect with previous work, which shows that the mean values of local densities evolve according to the hydrodynamic equations. Furthermore, the fact that there is essentially only one history with non-zero probabilty means that there is approximate decoherence.

Going beyond the assumption of a local equilibrium state, is however, much more difficult. We were able to see how this sort of state arises asymptotically in the simple diffusive model, but it is difficult to demonstrate in general. We gave some arguments for decoherence for more general initial states, but these still remain rather heuristic, and it would be useful to develop some explicit models in which decoherence via approximate conservation could be seen more clearly. This will be addressed in future publications.

\section{ACKNOWLEDGEMENTS}

I am deeply grateful to Todd Brun and Jim Hartle. Much of this work arose from many conversations with them over a long period of time.

\section{APPENDIX A: AN EHRENFEST}




\section{THEOREM FOR HISTORIES}

In this appendix, we give an alternative derivation of the result of Section $\mathrm{V}$, that the probabilities for histories of local densities are peaked about the evolution of the mean values (and hence about the hydrodynamic equations). There, the result relied on large $N$ statistics. Here, we show that it may be regarded as an example of a simple theorem for histories similar to the Ehrenfest theorem of elementary quantum mechanics. The result is as follows: for any Hamiltonian and any initial state, histories characterized by projections onto any operators are decoherent if the widths of the projections are taken to be sufficiently large, and the centers of the projections are also suitably chosen. Furthermore, the probabilities for these histories are then peaked about the time evolution of the expectation value of the operators in the given initial state.

At some level, this result must be almost trivial, since it is so very general ("any" initial state, "any" Hamiltonian, "any" operator). However, as we shall see, the hydrodynamic case considered in this paper is a situation to which it naturally applies, where it is little more than the statement that the quantum evolution is strongly concentrated around one particular history.

To give a flavour of the basic idea, consider the simplest possible case of a history characterized by a single projection at one moment of time. Such histories are trivially decoherent, and the probabity is given by the standard expression,

$$
p(\alpha)=\operatorname{Tr}\left(P_{\alpha} \rho\right)=\left\langle P_{\alpha}\right\rangle
$$

For simplicity, we will let the projection $P_{\alpha}$ be a Gaussian quasi-projector onto some operator $A$ with width $\sigma$ :

$$
P_{\alpha}=\frac{1}{\left(2 \pi \sigma^{2}\right)^{\frac{1}{2}}} \exp \left(-\frac{(A-\alpha)^{2}}{2 \sigma^{2}}\right)
$$


Gaussian projectors are exhaustive but only approximately exclusive.

Inserting this in (A1) and letting $\beta=\alpha-\langle A\rangle$, and $B=A-\langle A\rangle$, we have

$$
p(\alpha)=\frac{1}{\left(2 \pi \sigma^{2}\right)^{\frac{1}{2}}}\left\langle\exp \left(-\frac{(\beta-B)^{2}}{2 \sigma^{2}}\right)\right\rangle
$$

We are interested in the behaviour of this expression for large $\sigma$. Expanding for large $\sigma$ we obtain,

$$
p(\alpha)=\frac{1}{\left(2 \pi \sigma^{2}\right)^{\frac{1}{2}}} \exp \left(-\frac{\beta^{2}}{2 \sigma^{2}}\right)\left\langle 1+\frac{\beta B}{\sigma^{2}}-\frac{B^{2}}{2 \sigma^{2}}+\frac{\beta^{2} B^{2}}{2 \sigma^{4}}+O\left(\sigma^{-3}\right)\right\rangle
$$

(note that $\beta$ is taken to be of order $\sigma$ because of the Gaussian factor). Now, noting that $\langle B\rangle=0$, and $\left\langle B^{2}\right\rangle=(\Delta A)^{2}$, and reorganizing the result as an exponential, we find that for large $\sigma$,

$$
p(\alpha) \approx \frac{1}{\left(2 \pi\left(\sigma^{2}+(\Delta A)^{2}\right)\right)^{\frac{1}{2}}} \exp \left(-\frac{\left(\langle A\rangle-\alpha_{1}\right)^{2}}{2\left(\sigma^{2}+(\Delta A)^{2}\right)}\right)
$$

Therefore, for $\sigma^{2}>>(\Delta A)^{2}$, the probability is peaked about the expectation value of $A$. This is an intuitively expected result: a very broad sampling yields the average value. (The same qualitative result will probably also hold for exact projectors, but we do not consider this here).

The above result readily extends to non-trivial histories. First note that for Gaussian projectors, the time-dependent projectors may be written,

$$
P_{\alpha_{k}}\left(t_{k}\right)=\frac{1}{\left(2 \pi \sigma^{2}\right)^{\frac{1}{2}}} \exp \left(-\frac{\left(A_{t_{k}}-\alpha_{k}\right)^{2}}{2 \sigma^{2}}\right)
$$

where

$$
A_{t_{k}}=e^{i H\left(t_{k}-t_{0}\right)} A e^{-i H\left(t_{k}-t_{0}\right)}
$$

Then inserting in the expression for the probabilities (setting aside for the moment the question of decoherence) and expanding for large $\sigma$, the leading order behaviour is,

$$
p\left(\alpha_{1}, \alpha_{2}, \cdots \alpha_{n}\right)=\prod_{k=1}^{n} \exp \left(-\frac{\left(\left\langle A_{t_{k}}\right\rangle-\alpha_{k}\right)^{2}}{\sigma^{2}}\right)
$$


For simplicity, we have ignored normalization factors. Also, we have kept only the leading order terms for $\sigma^{2}>>\left(\Delta A_{t_{k}}\right)^{2}$ (for times $t_{k}$ ). The next correction may be computed, at some length, but the qualitative conclusion is the same.

Eq.(A8) indicates that the probabilites for histories are peaked about the evolution of the expectation value, $\left\langle A_{t}\right\rangle$. It is the natural generalization of (A5): the broadest possible sampling of a history, yields the evolution of the expectation value.

The result for the single time case extends easily to the multi-time case even though the $A_{t_{k}}$ 's do not commute, because their non-commutativity only enters at higher orders for large $\sigma$, and does not affect the configuration $\left\langle A_{t_{k}}\right\rangle$ about which the probabilities are peaked. For the same reason, one can also project onto a number of different noncommuting operators at each moment of time, as one needs to in the hydrodynamic case (see (5.7)-(5.9)), and still obtain a result of the form (A8).

The argument for decoherence is similar to those in Sections IV and V - it arises because there is essentially only one history with non-zero probability. More precisely, define a set containing just two histories as follows. Take the first history to be that characterized by projections in which the $\alpha_{k}$ 's are chosen to be very close to $\left\langle A_{t_{k}}\right\rangle$, and the width $\sigma^{2}$ is much greater than $(\Delta A)^{2}$. Then the probability for his history, which we denote simply $p$, will be very close to 1 . Then take the remaining history to be the complement of the first one. That is, the combination of all histories defined by projections in which at least one of the $\alpha_{k}$ 's is much greater than the width $\sigma$ away from $\left\langle A_{t_{k}}\right\rangle$. Then the probabilities for the second history, denote it $\bar{p}$, will be close to zero, since at least one of the projections will always pick up an exponentially small tail. There will therefore be approximate consistency, since the probabilites approximately add up to 1. (Equivalently, the decoherence functional is very small, since $|D|^{2}<p \bar{p}$.) 
As stated at the beginning, the above result is very general to the point of being almost vacuous. But it is useful in the hydrodynamic case because the local densities do have extremely small variances $(\Delta A)^{2}$ (compared to $\left.\langle A\rangle^{2}\right)$ in the local equilibrium state, so the widths $\sigma$ do not need to be taken to be unreasonably large for the conditions of the theorem to be satisfied. Hence we have provided an alternative proof that the probabilities for histories of local densities are peaked about the hydrodynamic equations.

\section{REFERENCES}

1. R.B.Griffiths, J.Stat.Phys. 36, 219 (1984); Phys.Rev.Lett. 70, 2201 (1993).

2. M.Gell-Mann and J.B.Hartle, in Complexity, Entropy and the Physics of Information, SFI Studies in the Sciences of Complexity, Vol. VIII, W. Zurek (ed.) (Addison Wesley, Reading, 1990); and in Proceedings of the Third International Symposium on the Foundations of Quantum Mechanics in the Light of New Technology, S. Kobayashi, H. Ezawa, Y. Murayama and S. Nomura (eds.) (Physical Society of Japan, Tokyo, 1990).

3. M.Gell-Mann and J.B.Hartle, Phys.Rev. D47, 3345 (1993).

4. R. Omnès, J.Stat.Phys. 53, 893 (1988). 53, 933 (1988); 53, 957 (1988); 57, 357 (1989); Ann.Phys. 201, 354 (1990); Rev.Mod.Phys. 64, 339 (1992).

5. H. F. Dowker and J. J. Halliwell, Phys. Rev. D46, 1580 (1992).

6. R.P.Feynman and F.L.Vernon, Ann. Phys. 24, 118 (1963).

7. A.Caldeira and A.Leggett, Physica 121A, 587 (1983). 
8. E.Joos and H.D.Zeh, Z.Phys. B59, 223 (1985).

9. See for example, J.P.Paz and W.H.Zurek, Phys.Rev.D48, 2728 (1993); W.Zurek, in Physical Origins of Time Asymmetry, edited by J.J.Halliwell, J.Perez-Mercader and W.Zurek (Cambridge University Press, Cambridge, 1994); W.Zurek, "Decoherence, Einselection, and the Existential Interpretation (The Rough Guide)", preprint quantph/9805065.

10. J.J.Halliwell and A.Zoupas, Phys.Rev. D52, 7294 (1995); D55, 4697 (1997).

11. J. B. Hartle, R. Laflamme and D. Marolf, Phys.Rev.D51, 7007 (1995).

12. The standard derivation of the hydrodynamic equations from their underlying microscopic origins is described in many places. An interesting discussion of the general issues is by G.E.Uhlenbeck, in the Appendix of, Lectures in Applied Mathematics: Probability and Related Topics in the Physics Sciences, by M.Kac (Interscience, NY, 1957). A useful basic discussion may be found in K.Huang, Statistical Mechanics, 2nd edition (New York, Chichester, Wiley, 1987). See also, for example, R.Balescu, Statistical Dynamics: Matter out of Equilibrium, (Imperial College Press, London, 1997); D. Forster, Hydrodynamic Fluctuations, Broken Symmetry and Correlation Functions (Benjamin, Reading, MA, 1975); H.J.Kreuzer, Nonequilibrium Thermodynamics and its Statistical Foundations (Clarendon Press, Oxford, 1981); D.N.Zubarev, Nonequlibrium Statistical Thermodynamics (Consultants Bureau, New York, 1974); D.Zubarev, V.Morozov and G.Röpke, Statistical Mechanics of Nonequilibrium Processes, Vol.1 (Akademie Verlag, Berlin, 1996).

13. J. B. Hartle, "Quasiclassical Domains in a Quantum Universe", preprint gr-qc/9404017 (1994). 
14. J.J.Halliwell, in Fundamental Problems in Quantum Theory, edited by D.Greenberger and A.Zeilinger, Annals of the New York Academy of Sciences, Vol 775, 726 (1994).

15. T.Brun and J.J.Halliwell, Phys.Rev. 54, 2899 (1996).

16. T.Brun and J.B.Hartle, "Hydrodynamic Equations from Decoherent Histories", Santa Barbara preprint (in preparation).

17. E. Calzetta and B. L. Hu, in Directions in General Relativity, edited by B. L. Hu and T. A. Jacobson (Cambridge University Press, Cambridge, 1993).

18. B.Carazza, "On the spatial density matrix for the centre of mass of a one-dimensional perfect gas", preprint quant-ph/9711005 (1997).

19. J.C.Flores, "Decoherence from internal degrees of freedom for clusters of mesoparticles: a hierachry of master equations", preprint quant-ph/9803032 (1998).

20. C.Anastopoulos, "Hydrodynamic equations from quantum field theory", preprint grqc/9805074 (1998).

21. R.Omnès, comment after talk by W.Zurek in Physical Origins of Time Asymmetry, edited by J.J.Halliwell, J.Perez-Mercader and W.Zurek (Cambridge University Press, Cambridge, 1994), page 209.

22. C.L.Feffermann, Bull.Amer.Math.Soc 9, 129 (1983).

23. H.F.Dowker and A.Kent, J.Stat.Phys. 82, 1575 (1996); Phys.Rev.Lett. 75, 3038 (1995).

24. I.Giardina and A.Rimini, Found.Phys. 26, 973 (1996).

25. R.B.Griffiths, Phys.Rev. A54, 2759 (1996); A57, 1604 (1998). 
26. J.B.Hartle, "Quantum Pasts and the Utility of Histories", preprint gr-qc/9712001.

27. H.Risken, The Fokker-Planck Equations: Methods of Solution and Applications, Second Edition (Springer-Verlag, Berlin,1989).

28. R.Omnès, Phys.Rev.A56, 3383 (1997).

29. L.Diósi, preprint gr-qc/9403046.

30. C.Anastopoulos and J.J.Halliwell, Phys.Rev. D51, 6870 (1995).

31. D.Finkelstein, Trans.N.Y.Acad.Sci. 25, 621 (1963); N.Graham, in The Many Worlds Interpretation of Quantum Mechanics, B.S.DeWitt and N.Graham (eds.) (Princeton University Press, Princeton, 1973); J.B.Hartle, Am.J.Phys. 36, 704 (1968). See also, E.Farhi, J.Goldstone and S.Gutmann, Ann.Phys.(NY) 192, 368 (1989).

32. The approach to equilibrium (or local equilibrium) is discussed in many places. Some examples are, G.S.Agarwal, Phys.Rev.A3, 838 (1971); M.A.Huerta and H.S.Robertson, J.Stat.Phys. 1, 393 (1969); M.Tegmark and H.S.Shapiro, Phys.Rev.E50, 2538 (1994).

33. D.N.Zubarev, Nonequilibrium Statistical Thermodynamics (Consultants Bureau, New York, 1974); 